\title{
Anticoncentration versus the Number of Subset Sums
}

\author{
Vishesh Jain Ashwin Sah* Mehtaab Sawhney ${ }^{\dagger}$
}

Received 19 January 2021; Published 28 June 2021

\begin{abstract}
Let $\vec{w}=\left(w_{1}, \ldots, w_{n}\right) \in \mathbb{R}^{n}$. We show that for any $n^{-2} \leq \varepsilon \leq 1$, if $\#\left\{\vec{\xi} \in\{0,1\}^{n}:\langle\vec{\xi}, \vec{w}\rangle=\tau\right\} \geq 2^{-\varepsilon n} \cdot 2^{n}$

for some $\tau \in \mathbb{R}$, then

$$
\#\left\{\langle\vec{\xi}, \vec{w}\rangle: \vec{\xi} \in\{0,1\}^{n}\right\} \leq 2^{O(\sqrt{\varepsilon} n)} .
$$

This exponentially improves the $\varepsilon$ dependence in a recent result of Nederlof, Pawlewicz, Swennenhuis, and Węgrzycki and leads to a similar improvement in the parameterized (by the number of bins) runtime of bin packing.
\end{abstract}

Key words and phrases: anticoncentration

\section{Introduction}

For $\vec{w}:=\left(w_{1}, \ldots, w_{n}\right) \in \mathbb{R}^{n}$ and a real random variable $\xi$, recall that the Lévy concentration function of $\vec{w}$ with respect to $\xi$ is defined for all $r \geq 0$ by

$$
\mathcal{L}_{\xi}(\vec{w}, r)=\sup _{\tau \in \mathbb{R}} \mathbb{P}\left[\left|w_{1} \xi_{1}+\cdots+w_{n} \xi_{n}-\tau\right| \leq r\right],
$$

where $\xi_{1}, \ldots, \xi_{n}$ are i.i.d. copies of $\xi$. In combinatorial settings (where $\vec{w} \in \mathbb{Z}^{n}$ ) a particularly natural and interesting case is when $r=0$ and $\xi$ is a Bernoulli random variable, i.e., $\xi=0$ with probability $1 / 2$ and $\xi=1$ with probability $1 / 2$. For lightness of notation, we will denote this special case by

$$
\rho(\vec{w})=\mathcal{L}_{\operatorname{Ber}(1 / 2)}(\vec{w}, 0)=\sup _{\tau \in \mathbb{R}} \mathbb{P}[\langle\vec{w}, \vec{\xi}\rangle=\tau] .
$$

In this note, we study the following question.

\footnotetext{
*Supported by NSF Graduate Research Fellowship Program DGE-1745302
}

${ }^{\dagger}$ Supported by NSF Graduate Research Fellowship Program DGE-1745302 
Question 1.1. For a vector $\vec{w}=\left(w_{1}, \ldots, w_{n}\right) \in \mathbb{R}^{n}$ with $\rho(\vec{w}) \geq \rho$, how large can the range

$$
\mathcal{R}(\vec{w})=\left\{w_{1} \xi_{1}+\cdots+w_{n} \xi_{n}: \xi_{i} \in\{0,1\}\right\}
$$

be?

The two extremal examples here are $\vec{w}=(0,0, \ldots, 0)$, which corresponds to $\rho(\vec{w})=1,|\mathcal{R}(\vec{w})|=1$ and $\vec{w}=\left(1,10, \ldots, 10^{n-1}\right)$, which corresponds to $\rho(\vec{w})=2^{-n},|\mathcal{R}(\vec{w})|=2^{n}$. Motivated by these examples, one may ask if there is a smooth trade-off between $\rho(\vec{w})$ and $|\mathcal{R}(\vec{w})|$. This turns out not to be the case. Indeed, for any $\varepsilon>0$, Wiman [6] gave an example of a $\vec{w} \in \mathbb{Z}^{n}$ for which $|\mathcal{R}(\vec{w})| \geq 2^{(1-\varepsilon) n}$ and $\rho(\vec{w}) \geq 2^{-0.7447 n}$. At the other end of the spectrum, when $\rho(\vec{w}) \geq 2^{-\varepsilon n}$, the so-called inverse Littlewood-Offord theory [3-5] heuristically suggests that $\vec{w}$ is essentially contained in a low-rank generalized arithmetic progression of 'small' volume so that $|\mathcal{R}(\vec{w})|$ is also 'small'. However, the number of 'exceptional elements' in the inverse Littlewood-Offord theorems [3-5] is unfortunately too large to be able to rigorously establish such a statement.

Nevertheless, in a recent work on the parameterized complexity of the bin packing problem (see Section 1.1), Nederlof, Pawlewics, Swennenhuis and Wegrzycki [2] showed that for any $\varepsilon>0$,

$$
\rho(\vec{w}) \geq 2^{-\varepsilon n} \Longrightarrow|\mathcal{R}(\vec{w})| \leq 2^{\delta(\varepsilon) n}
$$

where

$$
\delta(\varepsilon)=O\left(\frac{\log \log \left(\varepsilon^{-1}\right)}{\sqrt{\log \left(\varepsilon^{-1}\right)}}\right) .
$$

In particular, $\delta(\varepsilon) \rightarrow 0$ as $\varepsilon \rightarrow 0$. Moreover, we must have $\delta(\varepsilon) \geq(2-o(1)) \varepsilon$, as can be seen by considering

$$
\vec{w}=\left(C_{1}, \ldots, C_{1}, C_{2}, \ldots, C_{2}, \ldots, C_{n / k}, \ldots, C_{n / k}\right) \in \mathbb{R}^{n},
$$

where each $C_{i}$ is repeated $k$ times, and $C_{i}$ is sufficiently small compared to $C_{i+1}$ for all $i$. Indeed, for such $\vec{w}$, we have $\rho(\vec{w})=2^{-\left(\frac{1}{2}+o_{k}(1)\right) \frac{n}{k} \log _{2} k}$ while $|\mathcal{R}(\vec{w})| \leq 2^{\left(1+o_{k}(1)\right) \frac{n}{k} \log _{2} k}$.

We conjecture that this example is essentially the worst possible, so that $\delta(\varepsilon) \leq 2 \varepsilon$. We are able to show that

$$
\delta(\varepsilon)=O(\sqrt{\varepsilon})
$$

thereby obtaining an exponential improvement over (1.1). More precisely,

Theorem 1.2. Let $\varepsilon>0$. For any $n \geq \varepsilon^{-1 / 2}$ and any $\vec{w} \in \mathbb{R}^{n}$ satisfying $\rho(\vec{w}) \geq \exp (-\varepsilon n)$, we have

$$
|\mathcal{R}(\vec{w})| \leq \exp \left(C_{1.2} \varepsilon^{1 / 2} n\right)
$$

where $C_{1.2}$ is an absolute constant.

We prove this theorem in Section 2. 


\subsection{Application to bin packing}

The bin packing problem is a classic NP-complete problem whose decision version may be stated as follows: given $n$ items with weights $w_{1}, \ldots, w_{n} \in[0,1]$ and $m$ bins, each of capacity 1 , is there a way to assign the items to the bins without violating the capacity constraints? Formally, is there a map $f:[n] \rightarrow[m]$ such that $\sum_{i \in f^{-1}(j)} w_{i} \leq 1$ for all $j \in[m]$ ?

Björklund, Husfeldt, and Koivisto [1] provided an algorithm for solving bin packing in time $\tilde{O}\left(2^{n}\right)$ where the tilde hides polynomial factors in $n$. It is natural to ask whether the base of the exponent may be improved at all i.e. is there a (possibly randomized) algorithm to solve bin packing in time $\tilde{O}\left(2^{(1-\varepsilon) n}\right)$ for some absolute constant $\varepsilon>0$ ?

In recent work, Nederlof, Pawlewics, Swennenhuis and Wegrzycki [2] showed that this is true provided that the number of bins $m$ is fixed. More precisely, they showed that there exists a function $\sigma: \mathbb{N} \rightarrow \mathbb{R}^{>0}$ and a randomized algorithm for solving bin packing which, on instances with $m$ bins, runs in time $\tilde{O}\left(2^{(1-\sigma(m)) n}\right)$, where $\tilde{O}$ hides polynomials in $n$ as well as exponential factors in $m$. Their analysis, which crucially relies on (1.1), gives a very small value of $\sigma(m)$ satisfying

$$
\sigma(m) \leq 2^{-m^{9}} .
$$

Using Theorem 1.2 instead of (1.1) in a black-box manner in the analysis of [2], we exponentially improve the bound on $\sigma(m)$.

Corollary 1.3. With notation as above, the randomized algorithm of [2] solves bin packing instances with $m$ bins in time $\tilde{O}\left(2^{(1-\sigma(m)) n}\right)$ with high probability, for $\sigma: \mathbb{N} \rightarrow \mathbb{R}^{>0}$ satisfying

$$
\sigma(m)=\tilde{\Omega}\left(m^{-12}\right),
$$

where $\tilde{\Omega}$ hides logarithmic factors in $\mathrm{m}$.

Remark. This follows by noting that the function $f_{C}(m)$ in [2, Section 3.6] is $\tilde{\Theta}\left(m^{-2}\right)$ so that $\delta$ in [2, Section 3.6] is $\tilde{\Theta}\left(\mathrm{m}^{-3}\right)$. With Theorem 1.2, the function $\varepsilon(\delta)$ in the runtime analysis of [2, Section 3.4] satisfies $\varepsilon(\delta)=O\left(\delta^{2}\right)$. Therefore, the function $f_{B}(\delta)$ in the same section is $\tilde{O}\left(\delta^{4}\right)$, which is $\tilde{\Omega}\left(m^{-12}\right)$. Note that if one were able to establish the conjecturally optimal bound $\delta=O(\varepsilon)$, this would lead to $f_{B}(\delta)=\tilde{O}\left(\delta^{2}\right)$, thereby giving the quadratically better $\sigma(m)=\tilde{\Omega}\left(m^{-6}\right)$.

\subsection{Notation}

We use big- $O$ notation to mean that an absolute multiplicative constant is being hidden. We use $\operatorname{Ber}(1 / 2)$ to denote the balanced $\{0,1\}$ Bernoulli distribution and $\operatorname{Bin}(k)$ to denote the binomial distribution on $k$ trials with parameter $1 / 2$. Recall that $\operatorname{Bin}(k)$ is the sum of $k$ independent $\operatorname{Ber}(1 / 2)$ random variables. Given a distribution $\mu$, we let $\mu^{\otimes n}$ denote the distribution of a random vector with $n$ independent samples from $\mu$ as its coordinates. We also use the following standard additive combinatorics notation: $C+D=\{c+d: c \in C, d \in D\}$ is the sumset (if $C, D$ are subsets of the same abelian group), and for a positive integer $k$, we let $k \cdot C=C+\cdots+C$ ( $k$ times) be the iterated sumset. Finally, in some cases we will use the notation $\Sigma \cdot$ or $\int \cdot$ to denote that the expression in the sum or integral is the same as in the previous line to simplify the presentation of long expressions. 


\subsection{Outline of the proof}

As in [2], the starting point of our proof is the following observation: let $A$ denote a fixed (but otherwise arbitrary) set of unique preimages for points in $\mathcal{R}(\vec{w})$ (hence, $|A|=|\mathcal{R}(\vec{w})|$ ) and let $B$ denote the the set of preimages of a value $\tau \in \mathbb{R}$ realising $\rho(\vec{w})$. Then (Lemma 2.2) for any $k \geq 1$, the map $A \times(k \cdot B) \rightarrow A+k \cdot B$

is a bijection. In particular, if $\vec{a}$ is sampled from the uniform distribution on $A$ and $\vec{b}_{1}, \ldots, \vec{b}_{k}$ are independently sampled from the uniform distribution on $B$, then

$$
\begin{aligned}
|A| & =|A| \cdot \mathbb{P}\left[\vec{a}+\vec{b}_{1}+\cdots+\vec{b}_{k} \in\{0, \ldots, k+1\}^{n}\right] \\
& =|A| \cdot \sum_{\vec{x} \in\{0, \ldots, k+1\}^{n}} \mathbb{P}\left[\vec{a}+\vec{b}_{1}+\cdots+\vec{b}_{k}=\vec{x}\right] \\
& \leq|A| \cdot \sum_{\vec{x} \in\{0, \ldots, k+1\}^{n}} \mathbb{P}[\vec{a}=\vec{a}(\vec{x})] \cdot \mathbb{P}\left[\vec{b}_{1}+\cdots+\vec{b}_{k}=\vec{x}-\vec{a}(\vec{x})\right] \\
& \leq \sum_{\vec{x} \in\{0, \ldots, k+1\}^{n}} \mathbb{P}\left[\vec{b}_{1}+\cdots+\vec{b}_{k}=\vec{x}-\vec{a}(\vec{x})\right]
\end{aligned}
$$

In [2], the largeness of $B$ is exploited by finding, for every $a \in A$, a large subset of $B$ which is 'balanced' (in a certain sense) with respect to $a$. Instead, we exploit the largeness of $B$ directly by using the observation that the density of the uniform measure on $B$ with respect to the uniform measure on $\{0,1\}^{n}$ is at most $2^{n} /|B| \leq 2^{\varepsilon n}$. In particular, if we let $\mu_{k}$ denote the measure on $k \cdot B$ induced by the product measure on $B \times \cdots \times B$ via the map $\left(b_{1}, \ldots, b_{k}\right) \mapsto b_{1}+\cdots+b_{k}$ and if we let $\operatorname{Bin}(k)^{\otimes n}$ denote the $n$-fold product of the $\operatorname{Binomial}(k, 1 / 2)$ distribution, then the density of $\mu_{k}$ with respect to $\operatorname{Bin}(k)^{\otimes n}$ is at most $2^{k \varepsilon n}$. This allows us to replace the measure $\mu_{k}$ appearing in the last line of the above equation by $\operatorname{Bin}(k)^{\otimes n}$, at the cost of a factor of $2^{k \varepsilon n}$. Thus,

$$
|A| \leq 2^{k \varepsilon n} \cdot \sum_{\vec{x} \in\{0, \ldots, k+1\}^{n}} \mathbb{P}_{\vec{x} \sim \operatorname{Bin}(k)^{\otimes n}}[\vec{x}-\vec{a}(\vec{x})]
$$

The above expression is still complicated by the presence of the shift $\vec{a}(\vec{x})$, about which we have no information except that it lies in the set $A$. The key technical lemma in the proof is Lemma 2.1, which essentially allows us to remove this shift after paying a factor which depends on $|A|$. Ultimately, this gives an upper bound on the sum in terms of $|A|$ and $k$, which amounts to an upper bound on $|A|$ in terms of $k, \varepsilon$, and $|A|$. Optimizing the value of the free parameter $k$ now gives the desired conclusion.

\section{Proof of Theorem 1.2}

We begin by recording the following key comparison bound, which will be proved at the end of this section.

Lemma 2.1. Let $n \geq k \geq C_{2.1}$, where $C_{2.1}$ is a sufficiently large absolute constant and let $\delta>0$. For any $A \subseteq\{0,1\}^{n}$ with $|A| \leq \exp (\delta n)$, the following holds. Let $\vec{x}, \vec{b} \sim \operatorname{Bin}(k)^{\otimes n}$ be independent $n$-dimensional random vectors. Then,

$$
\mathbb{E}_{\vec{x}}\left[\sup _{\vec{a} \in A} \frac{\mathbb{P}_{\vec{b}}[\vec{b}=\vec{x}-\vec{a}]}{\mathbb{P}_{\vec{b}}[\vec{b}=\vec{x}]}\right] \leq \exp \left(C_{2.1}\left(\frac{1}{k}+\sqrt{\frac{\delta}{k}}\right) n\right)
$$


Let $n, \varepsilon$, and $\vec{w}$ be as in Theorem 1.2. Let $\tau$ be such that $\mathbb{P}[\langle\vec{w}, \vec{\xi}\rangle=\tau]=\rho(\vec{w})$, where $\vec{\xi}$ is a random vector with i.i.d. $\operatorname{Ber}(1 / 2)$ components. Let

$$
B=\left\{\vec{\xi} \in\{0,1\}^{n}:\langle\vec{w}, \vec{\xi}\rangle=\tau\right\} .
$$

In particular, $|B| \geq \exp (-\varepsilon n) \cdot 2^{n}$. Let $|\mathcal{R}(\vec{w})|=\exp (\delta n)$. For each $r \in \mathcal{R}(\vec{w})$, let $\vec{\xi}(r)$ be a fixed (but otherwise arbitrary) element of $\{0,1\}^{n}$ such that $\langle\vec{w}, \vec{\xi}(r)\rangle=r$. Let

$$
A=\left\{\vec{\xi}(r) \in\{0,1\}^{n}: r \in \mathcal{R}(\vec{w})\right\} .
$$

Note that, by definition, for any distinct $\vec{a}_{1}, \vec{a}_{2} \in A$, we have that $\left\langle\vec{w}, \vec{a}_{1}\right\rangle \neq\left\langle\vec{w}, \vec{a}_{2}\right\rangle$ and that $|A|=|\mathcal{R}(\vec{w})|=$ $\exp (\delta n)$.

We will make use of the simple, but crucial, observation from [2] that $A$ and $k \cdot B$ have a full sumset for all $k \geq 1$.

Lemma 2.2 ([2, Lemma 4.2]). The map $(\vec{a}, \vec{c}) \mapsto \vec{a}+\vec{c}$ from $A \times(k \cdot B)$ to $A+k \cdot B$ is injective.

Proof. Indeed, if $\vec{a}_{1}+\left(\vec{b}_{1}^{(1)}+\cdots+\vec{b}_{k}^{(1)}\right)=\vec{a}_{2}+\left(\vec{b}_{1}^{(2)}+\cdots+\vec{b}_{k}^{(2)}\right)$, where $\vec{a}_{i} \in A$ and $\vec{b}_{j}^{(i)} \in B$, then taking the inner product of both sides with $\vec{w}$ and using $\langle\vec{w}, \vec{b}\rangle=\tau$ for all $b \in B$, we see that $\left\langle\vec{w}, \vec{a}_{1}\right\rangle=\left\langle\vec{w}, \vec{a}_{2}\right\rangle$, which implies that $\vec{a}_{1}=\vec{a}_{2}$ by the definition of $A$.

We are now ready to prove Theorem 1.2.

Proof of Theorem 1.2. Let $k \geq 2$ be a parameter which will be chosen later depending on $\varepsilon$. We may assume $\varepsilon \in\left(0,\left(2 C_{2.1}\right)^{-2}\right)$ by adjusting $C_{1.2}$ appropriately at the end to make larger values trivial. By Lemma 2.2, for each $\vec{x} \in\{0, \ldots, k+1\}^{n}$ for which there exist $\vec{a} \in A$ and $\vec{c} \in k \cdot B$ with $\vec{a}+\vec{c}=\vec{x}$, there exists a unique such choice $\vec{a}=\vec{a}(\vec{x}) \in A$. (For $\vec{x} \notin A+k \cdot B$, we let $\vec{a}(\vec{x})$ be an arbitrary element of $A$.)

Now, let $\vec{a}$ be uniform on $A$, let $\vec{b}_{1}, \ldots, \vec{b}_{k}$ be uniform on $B$, and let $\vec{v}_{1}, \ldots, \vec{v}_{k}$ be uniform on $\{0,1\}^{n}$. Let $C_{i} \subseteq\{0, \ldots, k+1\}^{n}$ be the set of vectors with $i$ coordinates equal to $k+1$. For $\vec{x} \in\{0, \ldots, k+1\}^{n}$, we let $\vec{x}^{*} \in\{0, \ldots, k\}^{n}$ denote the vector obtained by setting every occurrence of $k+1$ in $\vec{x}$ to $k$. We have

$$
\begin{aligned}
1 & =\mathbb{P}\left[\vec{a}+\vec{b}_{1}+\cdots+\vec{b}_{k} \in\{0, \ldots, k+1\}^{n}\right] \\
& =\sum_{i=0}^{n} \sum_{\vec{x} \in C_{i}} \mathbb{P}\left[\vec{a}+\vec{b}_{1}+\cdots+\vec{b}_{k}=\vec{x}\right] \\
& \leq \sum_{i=0}^{n} \sum_{\vec{x} \in C_{i}} \mathbb{P}[\vec{a}=\vec{a}(\vec{x})] \mathbb{P}\left[\vec{b}_{1}+\cdots+\vec{b}_{k}=\vec{x}-\vec{a}(\vec{x})\right] \\
& \leq \frac{1}{|A|} \sum_{i=0}^{n} \sum_{\vec{x} \in C_{i}}\left(\frac{2^{n}}{|B|}\right)^{k} \mathbb{P}\left[\vec{v}_{1}+\cdots+\vec{v}_{k}=\vec{x}-\vec{a}(\vec{x})\right] \\
& \leq \frac{e^{k \varepsilon n}}{|A|} \sum_{i=0}^{n} \sum_{\vec{x} \in C_{i}} \mathbb{P}\left[\vec{v}_{1}+\cdots+\vec{v}_{k}=\vec{x}^{*}\right] \sup _{\vec{a} \in A} \frac{\mathbb{P}\left[\vec{v}_{1}+\cdots+\vec{v}_{k}=\vec{x}-\vec{a}\right]}{\mathbb{P}\left[\vec{v}_{1}+\cdots+\vec{v}_{k}=\vec{x}^{*}\right]} \\
& =\frac{e^{k \varepsilon n}}{|A|} \sum_{i=0}^{n}\left(1 / 2^{k}\right)^{i} \sum_{S \in\left(\begin{array}{c}
{[n]} \\
i
\end{array}\right)} \mathbb{E}_{\vec{x} \sim \operatorname{Bin}(k)^{\otimes([n] \backslash S)} \times\{k+1\}^{S}}\left[\sup _{\vec{a} \in A} \frac{\mathbb{P}\left[\vec{v}_{1}+\cdots+\vec{v}_{k}=\vec{x}-\vec{a}\right]}{\mathbb{P}\left[\vec{v}_{1}+\cdots+\vec{v}_{k}=\vec{x}^{*}\right]}\right] .
\end{aligned}
$$


Let $A_{S}$ be the set of elements in $A \subseteq\{0,1\}^{n}$ whose support contains $S$. Let

$$
A_{S}^{\prime}=\left\{\vec{a}^{\prime} \in\{0,1\}^{[n] \backslash S}: \exists \vec{a} \in A_{S} \text { with }\left.\vec{a}\right|_{[n] \backslash S}=\vec{a}^{\prime}\right\} .
$$

Recall that $|A|=\exp (\delta n)$. Abusing notation so that the supremum of an empty set is 0 , we can continue the above chain of inequalities to get that

$$
\begin{aligned}
1 & \leq \frac{e^{k \varepsilon n}}{|A|} \sum_{i=0}^{n}\left(1 / 2^{k}\right)^{i} \sum_{S \in\left(\begin{array}{c}
{[n]} \\
i
\end{array}\right)} \mathbb{E}_{\vec{x} \sim \operatorname{Bin}(k)^{\otimes([n] S)} \times\{k+1\}^{S}}\left[\sup _{\vec{a} \in A} \frac{\mathbb{P}\left[\vec{v}_{1}+\cdots+\vec{v}_{k}=\vec{x}-\vec{a}\right]}{\mathbb{P}\left[\vec{v}_{1}+\cdots+\vec{v}_{k}=\vec{x}^{*}\right]}\right] \\
& =\frac{e^{k \varepsilon n}}{|A|} \sum_{i=0}^{n}\left(1 / 2^{k}\right)^{i} \sum_{S \in\left(\begin{array}{c}
{[n]} \\
i
\end{array}\right)} \mathbb{E}_{\vec{x} \sim \operatorname{Bin}(k)^{\otimes([n] S)}}\left[\sup _{\vec{a} \in A_{S}^{\prime}} \frac{\left.\mathbb{P}\left[\vec{v}_{1}+\cdots+\vec{v}_{k}=(\vec{x}-\vec{a}) \times\{k\}^{S}\right]\right]}{\mathbb{P}\left[\vec{v}_{1}+\cdots+\vec{v}_{k}=\vec{x} \times\{k\} S\right]}\right] \\
& =\frac{e^{k \varepsilon n}}{|A|} \sum_{i=0}^{n}\left(1 / 2^{k}\right)^{i} \sum_{S \in\left(\begin{array}{c}
{[n]} \\
i
\end{array}\right)} \mathbb{E}_{\vec{\sim} \sim \operatorname{Bin}(k)^{\otimes([n] S S)}}\left[\sup _{\vec{a} \in A_{S}^{\prime}} \frac{\mathbb{P}\left[\left.\left(\vec{v}_{1}+\cdots+\vec{v}_{k}\right)\right|_{[n] \backslash S}=\vec{x}-\vec{a}\right]}{\mathbb{P}\left[\left.\left(\vec{v}_{1}+\cdots+\vec{v}_{k}\right)\right|_{[n] \backslash S}=\vec{x}\right]}\right] \\
& \leq \frac{e^{k \varepsilon n}}{|A|}\left(\sum_{i=0}^{n / 2} \cdot+\sum_{i=n / 2}^{n} 2^{-k i} \cdot 2^{n} \cdot\left(\max _{\ell} \frac{\max \left\{\left(\begin{array}{c}
k \\
\ell-1
\end{array}\right),\left(\begin{array}{l}
k \\
\ell
\end{array}\right)\right\}}{\left(\begin{array}{l}
k \\
\ell
\end{array}\right)}\right)^{n-i}\right) \\
& \leq \frac{e^{k \varepsilon n}}{|A|}\left(\sum_{i=0}^{n / 2} \cdot+n \cdot 2^{-k n / 2} \cdot 2^{n} \cdot k^{n}\right) \\
& \leq \frac{e^{k \varepsilon n}}{|A|}\left(\sum_{i=0}^{n / 2}\left(\begin{array}{c}
n \\
i
\end{array}\right) 2^{-k i} \exp \left(C_{2.1}\left(k^{-1}+(2 \delta)^{1 / 2} k^{-1 / 2}\right)(n / 2)\right)+2^{-k n / 4}\right) \\
& \leq \exp (-\delta n) \exp \left(O\left(k \varepsilon+k^{-1}+\delta^{1 / 2} k^{-1 / 2}\right) n\right)
\end{aligned}
$$

by Lemma 2.1 applied to $A_{S}$, as long as $n / 2 \geq k \geq C_{2.1} \geq 20$. To deduce the last line, note that $\left(\begin{array}{c}n \\ i\end{array}\right) 2^{-k i} \leq\left(2^{-k} e n / i\right)^{i}$, so for $i \geq\left\lceil e n / 2^{k-1}\right\rceil$ the sum of weighted binomials is bounded by a geometric series. Additionally, for $1 \leq i \leq e n / 2^{k}$, if this interval is nonempty, the sum of binomials is certainly bounded by $\exp \left(O\left(k^{-1} n\right)\right)$.

Hence, the above inequality yields

$$
\delta \leq C\left(k \varepsilon+k^{-1}+\delta^{1 / 2} k^{-1 / 2}\right)
$$

for some absolute constant $C>0$. Now letting $k=\varepsilon^{-1 / 2} / 2$ (note that this satisfies $2 C_{2.1} \leq 2 k=\varepsilon^{-1 / 2} \leq n$ ), we find that

$$
\delta=O\left(\varepsilon^{1 / 2}\right)
$$

as desired.

The proof of Lemma 2.1 relies on the following preliminary estimate.

Lemma 2.3. If $1 \leq s \leq k /(16 \pi)$, then

$$
\mathbb{E}_{x \sim \operatorname{Bin}(k)}\left(\frac{x}{k+1-x}\right)^{s} \leq \exp \left(10 \pi s^{2} / k\right)+2 k^{s}(4 / 5)^{k} .
$$


Proof. We let $x \sim \operatorname{Bin}(k)$ and $y=x-k / 2 \sim \operatorname{Bin}(k)-k / 2$ throughout. We let $z \sim \mathcal{N}(0, k \pi / 8)$. We have

$$
\begin{aligned}
\mathbb{E}_{x \sim \operatorname{Bin}(k)}\left[\left(\frac{x}{k+1-x}\right)^{s}\right] & =\mathbb{E}_{y}\left[\left(1+\frac{2 y-1}{k / 2+1-y}\right)^{s}\right] \\
& \leq \mathbb{E}_{y}\left[\left(1+\frac{2 y}{k / 2+1-y}\right)^{s} \mathbb{1}_{|y| \leq k / 3}\right]+k^{s} \mathbb{P}[|y| \geq k / 3] \\
& \leq \mathbb{E}_{y}\left[\left(1+\frac{2 y}{k / 2+1-y}\right)^{s} \mathbb{1}_{|y| \leq k / 3}\right]+2 k^{s}(4 / 5)^{k}
\end{aligned}
$$

Note that the probability estimate for $\mathbb{P}\left[\mathbb{1}_{|y| \geq k / 3}\right]$ follows from the sharp (entropy) version of the ChernoffHoeffding theorem. Since for $|y| \leq k / 3$,

$$
\frac{2 y}{(k / 2+1-y)} \leq \frac{2 y}{k / 2+1}+\frac{8 y^{2}}{(k / 2+1)^{2}}
$$

and using $(1+x) \leq \exp (x)$, we can continue the previous inequality as

$$
\begin{aligned}
\mathbb{E}_{x \sim \operatorname{Bin}(k)}\left[\left(\frac{x}{k+1-x}\right)^{s}\right] & \leq \mathbb{E}_{y}\left[\left(1+\frac{2 y}{k / 2+1}+\frac{8 y^{2}}{(k / 2+1)^{2}}\right)^{s} \mathbb{1}_{|y| \leq k / 3}\right]+2 k^{s}(4 / 5)^{k} \\
& \leq \mathbb{E}_{y}\left[\exp \left(\frac{4 s y}{k+2}+\frac{32 s y^{2}}{k^{2}}\right)\right]+2 k^{s}(4 / 5)^{k}
\end{aligned}
$$

Now, let $z_{1}, \ldots, z_{k}$ be i.i.d. $\mathcal{N}(0,1)$ random variables. Then,

$$
y \sim \frac{1}{2}\left(\operatorname{sgn} z_{1}+\cdots+\operatorname{sgn} z_{k}\right)
$$

Moreover, for any $-k \leq \ell \leq k$,

$$
\mathbb{E}\left[z_{1}+\cdots+z_{k} \mid \operatorname{sgn}\left(z_{1}\right)+\cdots+\operatorname{sgn}\left(z_{k}\right)=\ell\right]=\sqrt{\frac{2}{\pi}} \ell .
$$

In particular, under this coupling of $y, z_{1}, \ldots, z_{k}$, we have

$$
\mathbb{E}\left[z_{1}+\cdots+z_{k} \mid y\right]=\sqrt{\frac{8}{\pi}} y .
$$

Let $z=z_{1}+\cdots+z_{k}$, so that $z \sim \mathcal{N}(0, k)$. Then, by the convexity of

$$
f(y)=\exp \left(\frac{4 s y}{k+2}+\frac{32 s y}{k^{2}}\right)
$$


and using Jensen's inequality, we have

$$
\begin{aligned}
\mathbb{E}_{y} f(y) & =\mathbb{E}_{y, z_{1}, \ldots, z_{k}} f(y) \\
& =\mathbb{E}_{y, z_{1}, \ldots, z_{k}} f\left(\sqrt{\frac{\pi}{8}} \mathbb{E}[z \mid y]\right) \\
& \leq \mathbb{E}_{z} f(\sqrt{\pi} z / \sqrt{8}) \\
& =\mathbb{E}_{w \sim \mathcal{N}(0,1)} \exp \left(\frac{s \sqrt{2 k \pi}}{k+2} w+\frac{4 s \pi}{k} w^{2}\right) \\
& =\left(1-\frac{8 \pi s}{k}\right)^{-1 / 2} \exp \left(\frac{\pi s^{2} k^{2}}{(k+2)^{2}(k-8 \pi s)}\right) \\
& \leq \exp \left(\frac{8 \pi s}{k}+\frac{2 \pi s^{2}}{k}\right) \\
& \leq \exp \left(10 \pi s^{2} / k\right) .
\end{aligned}
$$

Finally, we can prove Lemma 2.1

Proof of Lemma 2.1. We may assume that $\delta \geq 2000 / k$ since the statement for $\delta<2000 / k$ follows from the statement for $\delta=2000 / k$. Also, note that we may assume that $\delta \leq \log 2$. For any $t \in \mathbb{R}$, we have

$$
\begin{aligned}
\mathbb{P}_{\vec{x}}\left[\sup _{\vec{a} \in A} \frac{\mathbb{P}_{\vec{b}}[\vec{b}=\vec{x}-\vec{a}]}{\mathbb{P}_{\vec{b}}[\vec{b}=\vec{x}]} \geq e^{t n}\right] & \leq|A| \sup _{\vec{a} \in A} \mathbb{P}_{\vec{x}}\left[\frac{\mathbb{P}_{\vec{b}}[\vec{b}=\vec{x}-\vec{a}]}{\mathbb{P}_{\vec{b}}[\vec{b}=\vec{x}]} \geq e^{t n}\right] \\
& \leq|A| \sup _{\vec{a} \in A} \inf _{s \geq 2} \exp (-s t n) \mathbb{E}_{\vec{x}}\left[\left(\frac{\mathbb{P}_{\vec{b}}[\vec{b}=\vec{x}-\vec{a}]}{\mathbb{P}_{\vec{b}}[\vec{b}=\vec{x}]}\right)^{s}\right] \\
& =|A| \sup _{\vec{a} \in A} \inf _{s \geq 2} \exp (-s t n) \prod_{i=1}^{n} \mathbb{E}_{x \sim \operatorname{Bin}(k)}\left[\left(\frac{\mathbb{P}\left[\operatorname{Bin}(k)=x-a_{i}\right]}{\mathbb{P}[\operatorname{Bin}(k)=x]}\right)^{s}\right] \\
& \leq|A| \inf _{s \geq 2} \exp (-s t n)\left(\mathbb{E}_{x \sim \operatorname{Bin}(k)}\left(\frac{x}{k+1-x}\right)^{s}\right)^{n} .
\end{aligned}
$$

In the last line, we have used that

$$
\begin{aligned}
\mathbb{E}_{x \sim \operatorname{Bin}(k)}\left[\left(\frac{x}{k+1-x}\right)^{s}\right] & \geq\left(\mathbb{E}_{x \sim \operatorname{Bin}(k)}\left[\frac{x^{2}}{(k+1-x)^{2}}\right]\right)^{s / 2} \\
& =\left(\sum_{\ell=0}^{k-1} \frac{\ell+1}{k-\ell}\left(\begin{array}{l}
k \\
\ell
\end{array}\right) 2^{-k}\right)^{s / 2} \\
& =\left(\sum_{\ell=0}^{k-1}\left(\frac{k+2}{k}+\frac{4(k+1)(\ell-k / 2)}{k^{2}}+\frac{(k+1)(k-2 \ell)^{2}}{k^{2}(k-\ell)}\right)\left(\begin{array}{l}
k \\
\ell
\end{array}\right) 2^{-k}\right)^{s / 2} \\
& \geq\left(\sum_{\ell=0}^{k-1}\left(\frac{k+2}{k}+\frac{4(k+1)(\ell-k / 2)}{k^{2}}\right)\left(\begin{array}{l}
k \\
\ell
\end{array}\right) 2^{-k}\right)^{s / 2}
\end{aligned}
$$


Anticoncentration versus the Number of Subset Sums

$$
\begin{aligned}
& =\left(\frac{k+2}{k}-\frac{3 k+4}{k} 2^{-k}\right)^{s / 2} \\
& \geq 1
\end{aligned}
$$

if $k \geq 3$. Therefore, by Lemma 2.3, we have

$$
\begin{aligned}
\mathbb{P}_{\vec{x}}\left[\sup _{\vec{a} \in A} \frac{\mathbb{P}_{\vec{b}}[\vec{b}=\vec{x}-\vec{a}]}{\mathbb{P}_{\vec{b}}[\vec{b}=\vec{x}]} \geq e^{t n}\right] & \leq|A| \inf _{s \geq 2} \exp (-s t n)\left(\mathbb{E}_{x \sim \operatorname{Bin}(k)}\left(\frac{x}{k+1-x}\right)^{s}\right)^{n} \\
& \leq|A| \inf _{2 \leq s \leq k /(16 \pi)} \exp (-s t n)\left(\exp \left(10 \pi s^{2} / k\right)+2 k^{s}(4 / 5)^{k}\right)^{n} \\
& \leq|A| \inf _{2 \leq s \leq k /(10 \log k)} \exp (-s t n)\left(\exp \left(12 \pi s^{2} / k\right)\right)^{n} \\
& \leq \begin{cases}|A| \exp \left(-\frac{k t^{2} n}{48 \pi}\right) & \text { if } \sqrt{\frac{96 \pi \delta}{k}} \leq t \leq(\log k)^{-1} \\
|A| \exp \left(-\frac{k n}{48 \pi(\log k)^{2}}\right) & \text { if }(\log k)^{-1} \leq t \leq \log k .\end{cases}
\end{aligned}
$$

Here, the second case follows by plugging in $s=k /(24 \pi \log k)$ and simplifying (assuming $C_{2.1}$ is large enough so $s \geq 2)$, and the first case follows from plugging in $s=k t /(24 \pi)$ which satisfies $2 \leq s \leq$ $k /(10 \log k)$ by the restriction on $t$ and $\delta$. Finally, since

$$
0 \leq \sup _{\vec{a} \in A} \frac{\mathbb{P}_{\vec{b}}[\vec{b}=\vec{x}-\vec{a}]}{\mathbb{P}_{\vec{b}}[\vec{b}=\vec{x}]} \leq\left(\max _{\ell} \frac{\max \left\{\left(\begin{array}{c}
k \\
\ell-1
\end{array}\right),\left(\begin{array}{l}
k \\
\ell
\end{array}\right)\right\}}{\left(\begin{array}{l}
k \\
\ell
\end{array}\right)}\right)^{n} \leq k^{n}
$$

we have

$$
\begin{aligned}
\mathbb{E}_{\vec{x}}\left[\sup _{\vec{a} \in A} \frac{\mathbb{P}_{\vec{b}}[\vec{b}=\vec{x}-\vec{a}]}{\mathbb{P}_{\vec{b}}[\vec{b}=\vec{x}]}\right]= & \int_{-\infty}^{\log k} \mathbb{P}\left[\sup _{\vec{a} \in A} \frac{\mathbb{P}_{\vec{b}}[\vec{b}=\vec{x}-\vec{a}]}{\mathbb{P}_{\vec{b}}[\vec{b}=\vec{x}]} \geq e^{t n}\right] n e^{t n} d t \\
\leq & \int_{1 / \log k}^{\log k} \cdot+\int_{\sqrt{96 \pi \delta / k}}^{1 / \log k}+\int_{-\infty}^{\sqrt{96 \pi \delta / k}} n e^{t n} d t \\
\leq & e^{\sqrt{96 \pi \delta / k} n}+\int_{\sqrt{96 \pi \delta / k}}^{1 / \log k}|A| \exp \left(-\frac{k t^{2} n}{48 \pi}\right) n e^{t n} d t \\
& +\int_{1 / \log k}^{\log k}|A| \exp \left(-\frac{k n}{48 \pi(\log k)^{2}}\right) n e^{t n} d t \\
\leq & \exp (O(\sqrt{\delta / k}) n)+\int_{\sqrt{96 \pi \delta / k}}^{1 / \log k} n e^{-t n} d t+1 \\
\leq & \exp (O(\sqrt{\delta / k}) n) .
\end{aligned}
$$




\section{Acknowledgments}

The authors are grateful to the anonymous reviewers for several suggestions which helped improve the presentation of the paper.

\section{References}

[1] Andreas Björklund, Thore Husfeldt, and Mikko Koivisto, Set partitioning via inclusion-exclusion, SIAM Journal on Computing 39 (2009), no. 2, 546-563. 3

[2] Jesper Nederlof, Jakub Pawlewicz, Céline M. F. Swennenhuis, and Karol Węgrzycki, A faster exponential time algorithm for bin packing with a constant number of bins via additive combinatorics, pp. 1682-1701. 2, 3, 4, 5

[3] Hoi Nguyen and Van Vu, Optimal inverse Littlewood-Offord theorems, Adv. Math. 226 (2011), no. 6, 5298-5319. MR 27759022

[4] Mark Rudelson and Roman Vershynin, The Littlewood-Offord problem and invertibility of random matrices, Advances in Mathematics 218 (2008), no. 2, 600-633. 2

[5] Terence Tao and Van H. Vu, Inverse Littlewood-Offord theorems and the condition number of random discrete matrices, Annals of Mathematics (2009), 595-632. 2

[6] Mårten Wiman, Improved constructions of unbalanced uniquely decodable code pairs, 2017, https: //www.diva-portal.org/smash/get/diva2:1120593/FULLTEXT01.pdf. 2

\section{AUTHORS}

Vishesh Jain

Department of Statistics, Stanford University

Stanford, California

visheshj@stanford.edu

https://jainvishesh.github.io

Ashwin Sah

Department of Mathematics, Massachusetts Institute of Technology

Cambridge, Massachusetts

asah@mit.edu

http://www.mit.edu/ asah/

Mehtaab Sawhney

Department of Mathematics, Massachusetts Institute of Technology

Cambridge, Massachusetts

msawhney@mit.edu

http://www.mit.edu/ msawhney/ 